\title{
Chemical Abundances of OB Stars with High Projected Rotational Velocities
}

\author{
Simone Daflon \\ Observatório Nacional, Rua General José Cristino 77 \\ CEP 20921-400, Rio de Janeiro BRAZIL \\ daflon@maxwell.on.br \\ Katia Cunha \\ Observatório Nacional, Rua General José Cristino 77 \\ CEP 20921-400, Rio de Janeiro BRAZIL \\ katia@gauss.on.br \\ Keith Butler \\ Institut für Astronomie und Astrophysik der Universität München, Scheinerstrasse 1, D-81679 \\ München, GERMANY \\ butler@usm.uni-muenchen.de \\ Verne V. Smith \\ Department of Physics, University of Texas at El Paso \\ El Paso, TX 79968-0515 USA \\ verne@barium.physics.utep.edu
}




\begin{abstract}
Elemental abundances of carbon, nitrogen, oxygen, magnesium, aluminum, and silicon are presented for a sample of twelve rapidly rotating OB $\operatorname{star}\left(v \sin i>60 \mathrm{kms}^{-1}\right)$ members of the Cep OB2, Cyg OB3 and Cyg OB7 associations. The abundances are derived from spectrum synthesis, using both LTE and non-LTE calculations. As found in almost all previous studies of OB stars, the average abundances are slightly below solar, by about 0.1 to 0.3 dex. In the case of oxygen, even with the recently derived low solar abundances the OB stars are closer to, but still below, the solar value. Results for the 9 Cep OB2 members in this sample can be combined with results published previously for 8 Cep OB2 stars with low projected rotational velocities to yield the most complete set of abundances, to date, for this particular association. These abundances provide a clear picture of both the general chemical and individual stellar evolution that has occurred within this association. By placing the Cep OB2 stars studied in an HR diagram we identify the presence of two distinct age subgroups, with both subgroups having quite uniform chemical abundances. Two stars are found in the older subgroup that show significant N/O overabundances, with both stars being two of the most massive, the most evolved, and most rapidly rotating of the members studied in Cep OB2. These characteristics of increased $\mathrm{N}$ abundances being tied to high mass, rapid rotation, and an evolved phase are those predicted from models of rotating stars which undergo rotationally driven mixing.
\end{abstract}

Subject headings: stars: abundances — stars: early-type — stars: $v \sin i$ 


\section{Introduction}

The determination of the chemical abundance distributions in stars often requires the identification and isolation of a suitable set of absorption lines whose individual line strengths, or equivalent widths, are well-defined and can be measured. These equivalent widths become the primary datasets upon which the abundance analyses of the stars are based. For early-type stars that have small values of $v \sin i$, defining a set of spectral lines whose equivalent widths can be measured accurately is usually a straightforward task, because their spectra are relatively free of blending lines. As rotational velocity increases and spectral line-widths broaden, however, a point is reached beyond which it becomes impossible to find adequate sets of unblended spectral lines on which to anchor a detailed abundance analysis. The distribution of projected rotational velocity, $v \sin i$, as a function of spectral type increases from F-G stars, where $v \sin i$ almost vanishes, to OB stars that typically rotate rapidly. Wolff et al. (1982) studied the distribution of rotational velocities in early-type stars and observed that, among hot stars, early B-type stars

show the lowest rotational velocities; $\langle v \sin i\rangle=110 \mathrm{kms}^{-1}$. However, even at the low end, these values of $v \sin i$ are high enough to blend the absorption lines to such an extent that prevents line identification and individual equivalent-width measurements. In this case, spectrum synthesis can be used to model the blended line profiles and extract elemental abundances.

Our previous studies of chemical abundances in OB stars - Daflon, Cunha \& Becker (1999) and Daflon et al. (2001), hereafter Papers I and II, respectively - analyzed only those observed targets with sharp lines $\left(v \sin i<60 \mathrm{kms}^{-1}\right)$ as the method adopted to derive elemental abundances was based on equivalent-width measurements. Due to this restriction, the final list of OB stars analyzed in these previous papers consisted of about $25 \%$ of the total number of observed targets for this project. However, many of the observed stars with relatively high $v \sin i$ still have conspicuous features in their spectra that can be compared with broadened synthetic line-profiles and thus provide information about their chemical compositions. This paper focuses on a subset of the observed sample OB stars having $v \sin i$ between roughly 60 and $140 \mathrm{kms}^{-1}$ with the intent being to derive stellar parameters and chemical abundances. Four stars are included from Paper I (HD 205948, HD 207951, HD 209339 and HD 239729 of the Cep OB2 association) and 3 stars from Paper II (HD 227696 and HD 228199 of Cyg OB3 and HD 202347 of Cyg OB7) for which stellar parameters were already derived but an abundance analysis was not done. In addition to these 7 stars, 5 other stars belonging to the Cep OB2 association are analyzed here. The addition of this set of rapidly rotating stars to those with lower projected rotational velocities analyzed already, enlarges our sample of observations for the current study of chemical abundances of OB stars in the Galactic disk as much as possible. In particular, this completes the sample for the study of the abundance distribution and investigation of possible inhomogeneities in the chemical composition of the gas that formed the main-sequence members (with spectral types between O9 and B3 and for which an abundance analysis can be done) of the Cep OB2 association.

In addition to their usefulness as probes of the general abundance distributions found in the Galactic disk and in $\mathrm{OB}$ associations, rapidly rotating B-stars can have their surface abundances 
altered by rotationally induced mixing. In a recent study, Heger \& Langer (2000) have presented surface abundance predictions for elements sensitive to mixing, such as boron, carbon, or nitrogen, in a set of stellar models of differing mass, rotational velocity, and age. The members of a typical OB association are all formed in the same place, but at slightly different times (with age differences of $\sim 10^{5}-10^{7} \mathrm{yrs}$ ), with differing masses and rotation velocities. Mass, age, and rotation are all variables that play a role in the degree of stellar mixing expected. In this paper certain elements sensitive to mixing, such as, $\mathrm{C}, \mathrm{N}$, and $\mathrm{O}$, are analyzed in Cep OB2 and the results can be used to test the rotating stellar models.

\section{Observations and Stellar Parameters}

The observations and data reduction are described in Papers I and II, however, a brief summary of the instruments follows. The data consist of spectra with a resolution of $\mathrm{R} \sim 60,000$, high signal-to-noise $(\mathrm{S} / \mathrm{N} \geq 150$ ), covering the spectral range from $4225-5285 \AA$ and obtained with the $2.1 \mathrm{~m}$ Otto M. Struve telescope plus Cassegrain Sandiford echelle spectrometer at the McDonald Observatory of the University of Texas. Additional spectra were obtained with the 2.7m Harlan J. Smith telescope, also at McDonald Observatory, and Coudé spectrometer having $\mathrm{R}=12,000$ and centered on the region of $H \gamma(4340 \AA)$.

In Paper I, an effective temperature scale for OB stars was derived using the reddening-free Q parameter from UBV photometry. The $\mathrm{T}_{\text {eff- }} \mathrm{Q}$ calibration, when combined with an analysis of the $\mathrm{H} \gamma$ line-profiles, has been found to be adequate for the derivation of the fundamental stellar parameters, $\mathrm{T}_{\text {eff }}$ and $\log \mathrm{g}$, needed to conduct an abundance analysis for the main-sequence $\mathrm{OB}$ stars. The adopted stellar parameters for the studied stars are collected in Table 1. Most of these are from Papers I and II and in this study we add the determinations for 5 rapidly rotating stellar members of the Cep OB2 association: HD235618, HD239681, HD239710, HD239745 and HD239748. All of the additional stellar parameters presented here were derived following the same approach adopted in Paper I. (See that paper for a more detailed discussion of the method). LTE plane-parallel model atmospheres were then calculated with the ATLAS9 code (Kurucz, 1991) for solar metallicity and a constant microturbulence velocity of $2 \mathrm{kms}^{-1}$. These model atmospheres were adopted for both the LTE and non-LTE abundance analyses.

\section{Analysis}

As a matter of consistency with our previous studies, we tried as much as possible to use the same sets of transitions of $\mathrm{C}, \mathrm{N}, \mathrm{O}, \mathrm{Si}, \mathrm{Mg}$ and $\mathrm{Al}$ selected in Papers I and II. However, because of the high rotational velocity of the target stars this was not always possible. In particular, the weak lines of C II, S III and Fe III become too shallow in stars with relatively high $v \sin i$. As a result of this, sulfur and iron abundances were not derived in this study. Because of the lack of $\mathrm{C}$ 
II lines, an attempt was made to derive carbon abundances using C III lines, as we are interested in complementing the sample for carbon as much as possible. The only possibility was to use the 3 strong C III lines in the region centered on $4650 \AA$, which were not used in our previous papers because these strong lines are very close to a set of O II transitions and are generally blended, even for stars that appear to rotate slowly. The selected spectral regions for this abundance study are gathered in Table 2 where, in the first column, we indicate the species that is dominant and whose abundance was adjusted in that particular region. Spectral lines used to construct linelists for the syntheses were taken from the Kurucz website (URL cfaku5.harvard.edu/) and the line gf-values were taken from the Opacity Project (OP), but also from Kurucz, when the atomic data needed was not available in the OP database.

\subsection{LTE Abundances}

LTE abundances of carbon, nitrogen, oxygen, magnesium, aluminum, and silicon were determined by fitting synthetic spectra calculated with the program LINFOR (originally developed by H. Holweger, M. Steffen, \& W. Steenbock) to the observed spectral regions containing the selected individual transitions. The synthetic profiles were then broadened for $v \sin i$ and limb darkening using a linear limb darkening coefficient interpolated from the values from table 2 in Wade \& Rucinski (1985). In addition, the synthetic spectra were broadened by the instrumental profile, while the macroturbulent velocity was set to zero (as suggested by Ebbets 1979 for main-sequence B-stars). As done in the abundance analyses of the sample of stars with low $v \sin i$, the $\mathrm{O}$ II transitions were used to derive microturbulent velocities as these lines are the most numerous in the sample and, most importantly, span a range in line-strength adequate to constrain the microturbulence. As a first step in this analysis, the O II line profiles were calculated for various values of microturbulence. The $v \sin i \mathrm{~s}$ in each case were allowed to vary and the best fit oxygen abundance for each profile was determined by means of a $\chi^{2}$-minimization. Figure 1 illustrates the type of diagram constructed in order to estimate the microturbulent velocity: this figure displays the behavior of the O II lines for the sample star HD239745, where best fit oxygen abundances (corresponding to seven spectral regions containing O II lines) are calculated for microturbulent velocities ranging from 2 to $10 \mathrm{kms}^{-1}$. Such a diagram is the equivalent of the usual 'abundance versus equivalent width' plot, where a solution is found when zero slope is obtained. By definition, the solution in Figure 1 will be the microturbulence value that yields approximately the same abundance for strong and weak lines: for this star a region of coincidence is found around a microturbulent velocity of $8.0 \mathrm{kms}^{-1}$.

Once a value for the microturbulence was established for each star, LTE synthetic spectra were calculated for all of the spectral regions listed in Table 2. Here again the $v \sin i$ s were left as free parameters and adjusted in each spectral synthesis and an individual projected rotational velocity was derived from each fit. The derived $v \sin i$ s for the individual fits did not, as expected, differ significantly and the average values are presented in the third column of Table 3 . The 
average LTE abundances for the studied elements, their standard deviations and the number [n] of fitted lines corresponding to each species are also found in Table 3. We stress that the carbon results in this table are for C III, while those in Papers I and II are from C II. For one star in the sample (the Cep OB2 member HD239745), however, it was possible to compare the C II and C III abudances as, for this star, the C II lines at $\lambda 5143.50 \AA, 5145.17 \AA$, and $5151.08 \AA$ were strong enough to be fitted: a carbon abundance of 8.22 provides a good fit for the $3 \mathrm{C}$ II lines, as well as the C III line whose abundance is listed in Table 3. Such an agreement between C II and C III abundances is not always achieved. In the literature, a few studies have pointed out that the $\mathrm{C}$ III lines at $4650 \AA$ usually provide abundances higher than the C II lines (e.g. Smartt et al. 1996, Rolleston et al. 1993). A discrepancy is also found from inspection of the results given in Gies \& Lambert (1992). In order to investigate systematic differences between C II and C III abundances, we decided to pursue an analysis of the C III lines in the stars analysed previously in Papers I and II and for which we published C II abundances. The results of the comparison will be discussed in Section 4.1.

The LTE abundances obtained follow trends found previously, namely that the elemental abundances in OB stars are, in general, sub-solar. However, a more detailed discussion must await results that take into account non-LTE effects. Non-LTE calculations for C, N, O and Si have already been presented in Papers I and II and proven to be small for the effective temperature and surface gravity range encompassed by the sample stars. Non-LTE synthetic profiles were calculated for each observed profile, and, in this study, new non-LTE calculations were added for two additional elements: $\mathrm{Mg}$ and $\mathrm{Al}$.

\subsection{Non-LTE abundances}

Non-LTE synthetic profiles were calculated using model atoms by Eber ( 1987 - C III), Becker \& Butler (1989 - N II), Becker \& Butler (1988 - O II), Dufton et al. (1986 - Al III), Przybilla et al. (2001- Mg II) and Becker \& Butler (1990 - Si III). The adopted model atoms contain most of the energy levels belonging to the main ionization stages with the addition of the lower levels of the adjacent stages with relevant populations in this temperature range. The completeness of the adopted model atoms is essential to guarantee an accurate ionization balance and the correct calculation of the level populations. The models include a number of permitted bound-bound transitions explicitly calculated, as well as transitions from levels fixed in LTE.

The level populations were computed with the program DETAIL, assuming LS-coupling to find the solution for the equations of statistical equilibrium and transfer; the line profiles were computed with Voigt profile functions using the program SURFACE. As a first step here, non-LTE synthetic profiles were fitted to the observed O II lines in order to determine the microturbulent velocity in a similar manner as done for the LTE analysis and discussed in Section 3.1. The microturbulence derived from O II lines for each star was then adopted to calculate the synthetic profiles for the species of the other studied elements. 
The left panels in Figure 2 show synthetic spectra calculated for the region between $\lambda 4412$ and $4419 \AA$ for HD239748. In this spectral region, the main contributions to the observed profiles correspond to the O II lines at $\lambda 4414 \AA$ and $4416 \AA$. In the top left panel five synthetic profiles are shown for different oxygen abundances of $\log \epsilon(\mathrm{O})=8.22,8.37,8.42,8.47$ and 8.62 and for $v \sin i$ of $65 \mathrm{~km}^{-1}$ (as indicated from the $\chi^{2}$-minimization shown in the bottom right panel.) The best fit oxygen abundance is represented by the synthetic profile with a solid line. It was derived via a $\chi^{2}$-minimization which is shown in the top right panel of this same figure. The bottom panel illustrates the sensitivity of the profiles to the variation of the projected rotational velocities, $v \sin i$, ranging, in this example, between 50 and $80 \mathrm{kms}^{-1}$.

Similarly to what was done in the LTE analysis, a best fit non-LTE abundance and $v \sin i$ value were obtained for each individually synthesized profile and Table 4 lists the average abundances and $v \sin i$ s for each target star. These are the final abundance results for $\mathrm{C}, \mathrm{N}, \mathrm{O}, \mathrm{Mg}, \mathrm{Al}$ and Si obtained in this study. The derived $v \sin i$ values had a small variation from fit-to-fit with standard deviations typically smaller than $10 \mathrm{kms}^{-1}$. Moreover, these $v \sin i$ s are very consistent with the $v \sin i$ s derived from the LTE syntheses. In order to investigate possible spurious trends due to systematic errors in the adopted $\mathrm{T}_{\text {eff }}$ scale, the non-LTE abundances obtained are plotted as a function of the effective temperatures in Figure 3: no major trends are found. The derived microturbulences in non-LTE are in all cases smaller than those derived in LTE, with the average being $2 \mathrm{~km} \mathrm{~s}^{-1}$ less. Since the microturbulence is an adhoc parameter needed in order to obtain an agreement between the abundances of strong and weak lines, it might be expected that a more realistic non-LTE treatment would reduce the need for microturbulence. This seems to be the case, but just slightly, as the derived microturbulences are still significant. However, this abundance analysis is based upon one dimensional plane-parallel model atmospheres in LTE and assume a constant microturbulent velocity. These models are still far from being able to represent the atmospheres of early-type stars perfectly and the microturbulence remains a required parameter in these calculations.

\subsection{Uncertainties}

The uncertainties in the adopted effective temperatures and surface gravities have been discussed in some detail in Paper I, with estimated errors of $4 \%$ in $\mathrm{T}_{\text {eff }}$ and of 0.1 dex in $\log g$. The uncertainty in the microturbulent velocity is assigned to be about $\pm 1.5 \mathrm{kms}^{-1}$. The final abundances are the non-LTE results and it is the non-LTE calculations that were used to investigate the final abundance uncertainties. Non-LTE synthetic profiles were calculated for new model atmospheres generated by adding the uncertainties to the adopted parameters for two stars in the sample. The elemental abundances derived from profile fitting are also subject to uncertainties in the continuum location as well as the choice of the projected rotational velocity. Based on the the variation of $\chi^{2}$ as a function of $v \sin i$, as shown on the bottom panel of Figure 3, an error of $7 \%$ is adopted due to $v \sin i$ and $5 \%$ due to continuum location. We note that variations 
in the linear limb darkening coefficient have negligible effects on both the derived values of $v \sin i$ and abundances. The resulting abundance uncertainties, as well as the total expected error $\left(\delta_{t}\right)$, are listed in Table 5. The total errors are $\sim 0.10-0.25$ dex, except for C III, which has a slightly larger error of $\sim 0.3$ dex that is dominated by the uncertainty in the effective temperature. The abundances of silicon and magnesium, based on strong lines, are very sensitive to the choice of the microturbulent velocity.

Previous abundances presented in Papers I and II were derived from equivalent width measurements. In order to investigate any systematic differences between abundances based on syntheses compared to those based on equivalent widths, all stars analyzed in both Papers I and II were re-analyzed using the synthesis techniques presented here. No significant offsets in the abundances were found, with differences typically less than 0.05 dex: both equivalent width and synthesis based abundances can be compared directly.

\section{Discussion}

\subsection{The General Abundance Trends}

It is usual to compare stellar abundance results with the abundances derived from the solar photosphere and meteorites. The Sun has been a reference point for obvious reasons. In this respect, several abundance studies of OB stars in the Galactic disk have noted the subsolar abundance values obtained for these early-type stars. With regard to this comparison, the non-LTE abundance results here indicate the same general pattern. For nitrogen and oxygen the derived target abundances are, in general, below the solar value. Some comments should be made concerning the accepted solar oxygen abundance. Two recent studies derive significantly lower solar $\mathrm{O}$ abundances than generally accepted earlier values, with Prieto, Lambert \& Asplund (2001) finding $\log \epsilon(\mathrm{O})=8.69$ and Holweger (2001) deriving 8.74. For the time being, we simply average these recent determinations and use $\log \epsilon(\mathrm{O})=8.72$ as the reference solar value. This new $\mathrm{O}$ abundance for the Sun also brings it closer to $\mathrm{O}$ abundances found in the ISM, with $\log \epsilon$ $(\mathrm{O})=8.43$ being the gas phase abundance as quoted by Sofia \& Meyer (2001): gas phase ISM

abundances for many elements are expected to be lower than stellar abundances for gas and stars from similar populations as some material in the ISM is in the form of grains. The lower solar abundances also result in better agreement with B stars in general, although these stars still fall below even this lower abundance. Subsolar abundances are also found for Al, with the exception of one star for which a solar $\mathrm{Al}$ abundance was derived. For $\mathrm{Mg}$ and $\mathrm{Si}$, it is found that four stars are above, or at, the solar abundance level, while the other stars lie below the solar abundance line. Figure 4 shows the non-LTE corrections for Mg II represented by $\Delta$ (LTE - non-LTE). These are always negative and can be as large as 0.25 dex. On the other hand, the LTE results would represent a very good approximation for aluminum, where the differences between LTE and non-LTE Al III abundances scatter around zero, with absolute values smaller than 0.08 dex 
(Figure 4). The corrections for nitrogen, oxygen and silicon are positive for all studied stars and typically smaller than 0.2 dex.

For carbon the abundance results are close to the solar value but these abundances need further discussion: for the 8 stars for which it was possible to obtain $\mathrm{C}$ abundances (derived in all cases solely from C III), 4 of them have carbon abundances that are very close (within 0.05 dex) to the solar value. We note that the derived carbon abundances here are in general closer to the solar abundance than other results in the literature, including our own in Papers I and II. This may be a systematic effect due to the choice of different lines and, in particular, different ionization stages: here strong C III lines and in Papers I and II the weaker C II transitions. Non-LTE C III corrections with respect to LTE abundances are not very large and range between -0.16 to +0.06 dex. These corrections, shown in Figure 4, are not able to bring the C II and C III into agreement. The non-LTE corrections for C II are typically smaller than 0.1 dex. A comparison of the C III and C II abundances was possible for 8 stars from Papers I and II. The derived results indicate that the LTE C III abundances are higher. For two stars we see very large discrepancies between the C III and C II results (both in LTE and non-LTE) of the order of 0.7-0.9 dex. Clearly further work is needed to clarify the situation and this is in progress.

Taken together, the mean non-LTE abundances for this sample of B stars still point to a general pattern of sub-solar abundances. Average abundances are $[\mathrm{C} / \mathrm{H}]=-0.15 \pm 0.15,[\mathrm{~N} / \mathrm{H}]=$ $-0.44 \pm 0.15,[\mathrm{O} / \mathrm{H}]=-0.22 \pm 0.14,[\mathrm{Mg} / \mathrm{H}]=-0.13 \pm 0.24,[\mathrm{Al} / \mathrm{H}]=-0.41 \pm 0.21$, and $[\mathrm{Si} / \mathrm{H}]=$ $-0.25 \pm 0.30$. The overall average abundance for all 6 elements, relative to solar, is $[\mathrm{m} / \mathrm{H}]=$ $-0.27 \pm 0.13$, or about a factor of 2 lower in metallicity than the Sun. Although subsolar, these abundances are still larger than most of the gas phase ISM abundances as summarized recently by Sofia \& Meyer (2001). Two elements, N and Si, are found to be below the gas phase ISM abundances. We note, however, that even for $\mathrm{N}$ in the Sun this seems to be marginally the case as well, while Si has large abundance uncertainties, as noted earlier.

\section{2. $\quad$ Cep OB2}

Nine stars of this sample belong to the Cep OB2 association, which has already been analyzed chemically in Paper I by means of equivalent-width measurements of spectral lines in sharp lined stars. The mean non-LTE abundances for a sample of 8 low $v \sin i$ stars are $\log \epsilon(\mathrm{C})=8.17 \pm 0.09$, $\log \epsilon(\mathrm{N})=7.62 \pm 0.10, \log \epsilon(\mathrm{O})=8.61 \pm 0.10$ and $\log \epsilon(\mathrm{Si})=7.21 \pm 0.28$. This study may now be complemented with the present sample, for which it is found to have mean non-LTE abundances of $8.37 \pm 0.15$ for carbon, $7.53 \pm 0.17$ for nitrogen, $8.47 \pm 0.14$ for oxygen and $7.28 \pm 0.33$ for silicon. The average abundances derived from both samples agree quite well for $\mathrm{N}, \mathrm{O}$ and $\mathrm{Si}$, despite the somewhat higher dispersions obtained for the sample of high $v \sin i$ stars. Carbon abundances are somewhat larger in this sample than in Paper I. However, as discussed before, carbon results in this paper are based on C III, instead of C II (as in Paper I): part of this difference may thus reside in systematic effects between $\mathrm{C}$ II and C III. If both samples are combined, then the entire 
set consists of 17 stars belonging to the Cep OB2 association and this represents $\sim 30$ percent of all the stars formed in the Cep OB2 association with spectral types between O9 and B3. These average abundances are then, relative to solar, $[\mathrm{C} / \mathrm{H}]=-0.26,[\mathrm{~N} / \mathrm{H}]=-0.40,[\mathrm{O} / \mathrm{H}]=-0.18$, and $[\mathrm{Si} / \mathrm{H}]=-0.30$ : all underabundant by roughly the same amount. The mean abundances confirm the results of Cep OB2 being slightly metal poor relative to the Sun by about 0.2-0.3 dex (the average underabundance of the four elements discussed here is -0.28).

With the addition of the sample of high $v \sin i$ stars, the number of Cep OB2 stars is twice as large as the number presented in Paper I and provides tighter constraints on the chemical distribution in the association. Cep OB2 is believed to be divided into two subgroups of different ages: Cep OB2a, with an estimated age of $7 \times 10^{6}$ years, extending over a large area within the association, and Cep OB2b, with an estimated age of $3 \times 10^{6}$ years, associated with the open cluster Trumpler 37 (Tr 37). It might be assumed that those stars assigned as members of $\operatorname{Tr} 37$ by Garmany \& Stencel (1992) belong to the younger subgroup (HD s 205794, 206183, 206267D, 207538, 239724, 205948, 239729, and 239748), while the rest of the sample (HD s 206327, 239742, 239743, 207951, 209339, 235618, 239681, 239710 and 239745) belongs to the older subgroup, Cep OB2a.

This assumption can be tested by using the derived stellar parameters to construct a variation of an HR-diagram for Cep OB2. The surface gravity (as $\log g$ ) is plotted versus $\log \mathrm{T}_{\text {eff }}$ and this is shown in the top panel of Figure 5 for all 17 Cep OB2 members in the sample. The open symbols correspond to members assigned to Tr 37 (and would be expected to be the younger stars of Cep OB2b), while the filled symbols are the other Cep OB2 stars. Stellar model tracks from Schaller et al. (1992) are shown as solid curves for $\mathrm{M}=7 \mathrm{M}_{\odot}, 9 \mathrm{M}_{\odot}, 12 \mathrm{M}_{\odot}, 15 \mathrm{M}_{\odot}$, and $20 \mathrm{M}_{\odot}$ models (with solar heavy-element abundances), while the dashed line shows the zero-age main sequence (ZAMS). It is clear from this form of an HR-diagram that the Cep OB2 members in this sample do generally segregate into two evolutionary groups, with a set of 10 stars falling very near the ZAMS, and a second, somewhat evolved group of 7 stars. The earlier assumption of assigning age status based on Tr 37 membership is a reasonable one: 7 of 8 stars identified as Tr 37 members fall on the ZAMS. Using the HR-diagram as the discriminator, 7 stars should be put into the older group (Cep OB2a: HD s 206327, 207951, 235618, 239681, 239724, 239742, and 239743), and 10 stars into the younger group (Cep OB2b: HD s 205794, 205948, 206183, 206267D, 207538, 209339, 239710, 239729, 239745, and 239748).

Abundances can be compared between the two subgroups of Cep OB2 (again using only the non-LTE abundances). Average abundances for Cep OB2a (the older subgroup) are $\log \epsilon(\mathrm{C})=$ $8.27 \pm 0.20, \log \epsilon(\mathrm{N})=7.66 \pm 0.08, \log \epsilon(\mathrm{O})=8.60 \pm 0.13$, and $\log \epsilon(\mathrm{Si})=7.28 \pm 0.32(\mathrm{Mg}$ and $\mathrm{Al}$ are not included in the subgroup comparison as non-LTE results are not available from Paper I). The corresponding average abundances for Cep OB2b are $\log \epsilon(\mathrm{C})=8.32 \pm 0.15, \log \epsilon(\mathrm{N})=$ $7.52 \pm 0.15, \log \epsilon(\mathrm{O})=8.49 \pm 0.14$, and $\log \epsilon(\mathrm{Si})=7.23 \pm 0.31$. The two subgroups have similar average abundances considering the abundance uncertainties, and this confirms the conclusions from Paper I that Cep OB2 is rather chemically homogeneous. 
The behavior of the $\mathrm{N} / \mathrm{O}$ abundance ratios are investigated more carefully in the bottom two panels of Figure 5, where the $\log (\mathrm{N} / \mathrm{O})$ abundances are plotted versus log g (middle panel) and $v \sin i$ (bottom panel). The abundance ratio of $\mathrm{N} / \mathrm{O}$ is shown, as the ratio is somewhat less sensitive to stellar parameter uncertainties, while surface gravity is used as a measure of evolutionary state (see the top panel of Figure 5), and projected rotational velocity is used to test for rotationally-induced mixing (Heger \& Langer 2000). In general, the N/O ratios of stars in both subgroups display small scatter, with $\log (\mathrm{N} / \mathrm{O})=-0.96 \pm 0.13$, however, two N-rich stars do stand out, especially in the middle panel as having lower values of $\log g$ (and are thus more evolved). These two stars are HD235618 and HD239681 and are Cep OB2a members. In the top panel of Figure 5, these N-rich stars are the two most evolved stars falling nearly on the $15 \mathrm{M}_{\odot}$ model track and also have two of the largest projected rotational velocities (bottom panel of Figure 5). It should be remembered, of course, that stars with low $v \sin i$ could also be rapid rotators viewed nearly pole-on, but stars with high $v \sin i$ values are rapid rotators, thus HD235618 and HD239681 are rotating rapidly. This suggests that rotation in fairly massive stars has led to mixing of CN-processed material to the surface. Nitrogen enhancements of nearly a factor of two are indicated. In a simple mixing scheme of only CN-processing, the sum of $\mathrm{C}$ plus $\mathrm{N}$ nuclei will be conserved; with an assumed starting abundance of $\log \epsilon(\mathrm{C})=8.35$ and $\log \epsilon(\mathrm{N})=7.55$, then an increase to $\log \epsilon(\mathrm{N})=7.80$ (as observed), would result in a carbon abundance change of only -0.08 dex: such a change in $\mathrm{C}$ would be lost in the noise of the $\mathrm{C}$-abundance uncertainties (especially in view of the possible differences between C II and C III abundances).

Heger \& Langer (2000) have computed models of rotating massive stars $\left(M=8-20 \mathrm{M}_{\odot}\right)$ that include surface abundance changes caused by rotationally induced mixing. They find that surface

${ }^{14} \mathrm{~N}$-abundances can increase by about a factor of 2 (as observed in two of the Cep OB2 members), in $12-20 \mathrm{M}_{\odot}$ stars that have rotational velocities greater than about $200 \mathrm{~km} \mathrm{~s}^{-1}$ and ages of $\sim 2-5$ Myr. The two Cep OB2 stars with $\mathrm{N}$-abundances enhanced by about +0.3 dex have masses close to $15 \mathrm{M}_{\odot}$, and ages of a few Myr. With projected rotational velocities of 100 and $140 \mathrm{~km} \mathrm{~s}^{-1}$, respectively, these two stars could easily have true rotational velocities of $200 \mathrm{~km} \mathrm{~s}^{-1}$ or larger. A test of the mixing hypothesis could be facilitated by a comparison of boron abundances in a sample of these stars.

\section{Conclusions}

Both the LTE and non-LTE abundances of C, N, O, Mg, Al, and Si derived here continue to indicate that young $\mathrm{O}$ and $\mathrm{B}$ stars in this part of the Galaxy have slightly subsolar abundances. The inclusion of C III lines in this analysis does, however, yield carbon abundances that are somewhat closer to solar than those abundances derived from C II lines.

With 9 Cep OB2 members in this sample, plus 8 members with low values of $v \sin i$ studied previously (Paper I), the chemical abundances in this association are now better defined and we have probed the chemical composition of the gas that formed the OB stars of this association with 
stellar masses roughly between $7-15 \mathrm{M}_{\odot}$ as much possible. Two distinct age subgroups within Cep OB2 can be identified based upon the stellar parameters derived here, with both subgroups having similar elemental abundances. Two N-rich stars are identified in the older subgroup, however, and these two stars are both rapidly rotating $\left(v \sin i \geq 100 \mathrm{~km} \mathrm{~s}^{-1}\right)$ and rather massive $\left(\mathrm{M} \sim 15 \mathrm{M}_{\odot}\right)$. Their nitrogen overabundances compare well to those predicted from stellar models by Heger \& Langer (2000) that include surface abundance changes brought about by rotationally induced mixing.

S.D. acknowledges a DAAD fellowship and the people at the Sternwarte, Munich. K.C. thanks David Lambert for travel support to observe at McDonald Observatory. V.V.S. acknowledges support from NASA (NAG5-9213) and the National Science Foundation (AST99-87374). 


\section{REFERENCES}

Becker, S. R. \& Butler, K. 1988, A\&A 201, 232

Becker, S. R. \& Butler, K. 1989, A\&A 209, 244

Becker, S. R. \& Butler, K. 1990, A\&AS 84, 95

Daflon, S., Cunha, K. \& Becker, S. 1999, ApJ 522, 950 (Paper I)

Daflon, S., Cunha, K., Becker, S. \& Smith, V. V. 2001, ApJ, 552, 309 (Paper II)

Dufton, P. L., Brown, P. J. F., Lennon, D. J. \& Lynas-Gray, A. E. 1986, MNRAS 222, 713

Ebbets, D. 1979, ApJ 227, 510

Eber, F. 1987, Diplomarbeit Munich

Garmany, C. D. \& Stencel, R. E. 1992, A\&AS 94, 211

Gies, D. \& Lambert, D. L. 1992, ApJ 387, 673

Grevesse, N., Noels, A. \& Sauval, A. J. 1996 in "Cosmic abundances" ed. S. S. Holt \& G. Sonneborn (San Francisco: ASP), 117

Heger, A., \& Langer, N. 2000, ApJ, 544, 1016

Holweger, H. 2001, in Solar and Galactic Composition, ed. R. F. Wimmer-Schweingruber (Berlin: Springer), in press

Kurucz, R.L., 1991, in: Crivellari L. et al (eds), Stellar Atmospheres: Beyond Classical Models, NATO ASI Ser. C-152, p 441

Prieto, C. A., Lambert, D. L., \& Asplund, M. 2001, ApJ, in press

Przybilla, N., Butler, K., Becker, S. R. \& Kudritzki, R.P. 2001, A\&A

Sofia, U. J., \& Meyer, D. M. 2001, ApJ, 554, L224

Schaller, G., Schaerer, D., Meynet, g., \& Maeder, A. 1992, A\&AS, 96, 269

Smartt, S. J., Dufton, P. L. \& Rolleston, W. R. J. 1996, A\&A 305, 164

Rolleston, W. R. J., Brown, P. J. F., Dufton, P. L. \& Fitzsimmons, A. 1993, A\&A 270, 107

Wade, R. A., Rucinski, S. M. 1985, A\&ASS, 60, 471

Wolff, S. C., Edwards, S. \& Preston, G. W. 1982, ApJ 252, 322 
Table 1. Atmospheric Parameters

\begin{tabular}{ccll}
\hline Star & Association & $\mathrm{T}_{\text {eff }}(\mathrm{K})$ & $\log g$ \\
\hline HD 202347 & Cyg OB7 & $23280^{b}$ & $4.13^{b}$ \\
HD 205948 & Cep OB2 & $24350^{a}$ & $4.25^{a}$ \\
HD 207951 & Cep OB2 & $20650^{a}$ & $3.88^{a}$ \\
HD 209339 & Cep OB2 & $31250^{a}$ & $4.28^{a}$ \\
HD 227696 & Cyg OB3 & $29100^{b}$ & $4.45^{b}$ \\
HD 228199 & Cyg OB3 & $29870^{b}$ & $4.45^{b}$ \\
HD 235618 & Cep OB2 & 27180 & 3.75 \\
HD 239681 & Cep OB2 & 26830 & 3.70 \\
HD 239710 & Cep OB2 & 21900 & 4.50 \\
HD 239729 & Cep OB2 & $28450^{a}$ & $4.22^{a}$ \\
HD 239745 & Cep OB2 & 27340 & 4.45 \\
HD 239748 & Cep OB2 & 27480 & 4.42 \\
\hline
\end{tabular}


TABle 2. Linelists

\begin{tabular}{|c|c|c|c|c|}
\hline Wavelength interval & $\lambda(\AA)$ & Species & $\chi(\mathrm{eV})$ & $\log (g f)$ \\
\hline $4234-4244$ & 4236.93 & N II & 23.24 & 0.39 \\
\hline \multirow[t]{6}{*}{ N II } & 4237.05 & N II & 23.24 & 0.56 \\
\hline & 4237.94 & O II & 28.83 & -0.99 \\
\hline & 4239.48 & O III & 33.15 & -2.04 \\
\hline & 4241.75 & N II & 23.24 & 0.22 \\
\hline & 4241.79 & N II & 23.25 & 0.72 \\
\hline & 4242.50 & $\mathrm{~N}$ II & 23.25 & -0.34 \\
\hline $4412-4419$ & 4413.11 & O II & 28.94 & -0.73 \\
\hline \multirow[t]{4}{*}{ O II } & 4414.88 & O II & 23.44 & 0.22 \\
\hline & 4416.97 & O II & 23.42 & -0.04 \\
\hline & 4417.10 & N II & 23.42 & -0.34 \\
\hline & 4418.84 & S III & 18.24 & -1.92 \\
\hline $4448-4455$ & 4448.34 & O II & 28.36 & 0.07 \\
\hline O II & 4452.38 & O II & 23.44 & -0.73 \\
\hline $4476-4484$ & 4479.88 & Al III & 20.78 & 0.90 \\
\hline \multirow{4}{*}{ Mg II, Al III } & 4479.97 & $\mathrm{Al} \mathrm{III}$ & 20.78 & 1.02 \\
\hline & 4481.13 & Mg II & 8.86 & 0.74 \\
\hline & 4481.15 & Mg II & 8.86 & -0.56 \\
\hline & 4481.33 & Mg II & 8.86 & 0.59 \\
\hline $4550-4554$ & 4552.41 & S II & 15.07 & -0.10 \\
\hline Si III & 4552.62 & Si III & 19.02 & 0.28 \\
\hline $4565-4570$ & 4567.84 & Si III & 19.02 & 0.06 \\
\hline \multirow[t]{2}{*}{ Si III } & 4569.06 & Ne II & 34.93 & 0.14 \\
\hline & 4569.26 & O III & 46.00 & 0.07 \\
\hline $4572-4576$ & 4574.42 & Ne II & 34.84 & -0.16 \\
\hline Si III & 4574.76 & Si III & 19.02 & -0.42 \\
\hline $4589-4593$ & 4591.01 & O II & 25.66 & 0.32 \\
\hline \multicolumn{5}{|l|}{ O II } \\
\hline $4605-4614$ & 4607.15 & $\overline{\mathrm{N}}$ II & 18.46 & -0.48 \\
\hline \multirow[t]{5}{*}{ N II, O II } & 4608.08 & N II & 23.48 & -0.25 \\
\hline & 4609.37 & O II & 29.07 & 0.71 \\
\hline & 4610.17 & O II & 29.06 & -0.17 \\
\hline & 4610.61 & O III & 45.94 & 0.02 \\
\hline & 4613.10 & O II & 29.07 & -0.59 \\
\hline $4628-4632$ & 4629.97 & C II & 24.79 & 0.50 \\
\hline \multirow[t]{4}{*}{ N II } & 4630.54 & N II & 18.48 & 0.09 \\
\hline & 4631.27 & Si IV & 36.42 & 0.85 \\
\hline & 4631.27 & Si IV & 36.42 & -0.58 \\
\hline & 4631.27 & Si IV & 36.42 & 0.96 \\
\hline $4637-4545$ & 4638.28 & $\overline{\text { Si III }}$ & 28.07 & -0.44 \\
\hline \multirow[t]{5}{*}{ N II, O II } & 4638.86 & O II & 22.97 & -0.35 \\
\hline & 4640.64 & N III & 30.46 & 0.14 \\
\hline & 4641.83 & O II & 22.98 & 0.05 \\
\hline & 4641.85 & N III & 30.46 & -0.81 \\
\hline & 4643.09 & N II & 18.48 & -0.39 \\
\hline $4647-4651$ & 4647.42 & C III & 29.54 & 0.06 \\
\hline \multirow[t]{6}{*}{ C III, O II } & 4647.59 & O II & 29.06 & -0.64 \\
\hline & 4649.14 & O II & 23.00 & 0.33 \\
\hline & 4650.25 & C III & 29.54 & -0.15 \\
\hline & 4650.85 & O II & 23.00 & -0.35 \\
\hline & 4651.02 & C III & 38.22 & -0.47 \\
\hline & 4651.47 & C III & 29.54 & -0.63 \\
\hline
\end{tabular}


TABle 2. Linelists (continued)

\begin{tabular}{clccc}
\hline Wavelength interval & $\lambda(\AA)$ & Species & $\chi(\mathrm{eV})$ & $\log (g f)$ \\
\hline $4658-4664$ & 4659.06 & C III & 38.22 & -0.69 \\
O II & 4661.64 & O II & 22.98 & -0.25 \\
& 4663.64 & C III & 38.22 & -0.57 \\
& 4665.86 & C III & 38.22 & 0.01 \\
\hline $4904-4908$ & 4904.78 & N III & 39.40 & -0.26 \\
O II & 4906.82 & O II & 26.30 & -0.05 \\
\hline $4939-4945$ & 4941.10 & O II & 26.55 & 0.07 \\
O II & 4943.00 & O II & 26.56 & 0.33 \\
\hline $5003-5012$ & 5005.15 & N II & 20.67 & 0.61 \\
N II & 5007.33 & N II & 20.94 & 0.17 \\
& 5010.62 & N II & 18.47 & -0.61 \\
\hline
\end{tabular}


TAble 3. LTE Abundances

\begin{tabular}{|c|c|c|c|c|c|c|c|c|}
\hline Star & $\begin{array}{c}\xi \\
\left(\mathrm{kms}^{-1}\right)\end{array}$ & $\begin{array}{c}v \sin i \\
\left(\mathrm{kms}^{-1}\right)\end{array}$ & $\log \epsilon(\mathrm{C})$ & $\log \epsilon(\mathrm{N})$ & $\log \epsilon(\mathrm{O})$ & $\log \epsilon(\mathrm{Mg})$ & $\log \epsilon(\mathrm{Al})$ & $\log \epsilon(\mathrm{Si})$ \\
\hline HD 202347 & 7.5 & $121 \pm 7$ & - & $7.67 \pm 0.10[3]$ & $8.62 \pm 0.11[5]$ & $7.15[1]$ & $6.30[1]$ & $7.28 \pm 0.04[3]$ \\
\hline HD 205948 & 7 & $146 \pm 12$ & $8.27[1]$ & $7.53 \pm 0.09[2]$ & $8.47 \pm 0.10[4]$ & $6.98[1]$ & $6.20[1]$ & $7.13 \pm 0.13[3]$ \\
\hline HD 207951 & 6.5 & $84 \pm 2$ & $8.53[1]$ & $7.76 \pm 0.03[3]$ & $8.75 \pm 0.09[5]$ & - & - & $7.18 \pm 0.06[3]$ \\
\hline HD 209339 & 7 & $101 \pm 9$ & - & $7.47 \pm 0.13[3]$ & $8.55 \pm 0.14[5]$ & $7.32[1]$ & $6.10[1]$ & $7.63 \pm 0.13[3]$ \\
\hline HD 227696 & 12 & $120 \pm 5$ & - & $7.58 \pm 0.09[2]$ & $8.62 \pm 0.11[5]$ & - & - & $7.61 \pm 0.14[3]$ \\
\hline HD 228199 & 8 & $105 \pm 5$ & $8.44[1]$ & $7.64 \pm 0.09[3]$ & $8.77 \pm 0.14[5]$ & $7.54[1]$ & $6.10[1]$ & $7.62 \pm 0.01[3]$ \\
\hline HD 235618 & 12 & $101 \pm 2$ & $8.60[1]$ & $7.90[1]$ & $8.67 \pm 0.11[4]$ & $7.33[1]$ & $6.00[1]$ & $7.95 \pm 0.14[2]$ \\
\hline HD 239681 & 10 & $140 \pm 10$ & $8.08[1]$ & $7.73 \pm 0.15[3]$ & $8.61 \pm 0.09[5]$ & $7.70[1]$ & $6.40[1]$ & $7.83 \pm 0.18[2]$ \\
\hline HD 239710 & 8 & $63 \pm 6$ & $8.40[1]$ & $7.62 \pm 0.07[3]$ & $8.63 \pm 0.09[5]$ & $7.44[1]$ & $5.95[1]$ & $7.88 \pm 0.20[3]$ \\
\hline HD 239729 & 6 & $100 \pm 7$ & - & $7.44 \pm 0.11[3]$ & $8.49 \pm 0.20[7]$ & $7.19[1]$ & $5.90[1]$ & $7.07 \pm 0.02[3]$ \\
\hline HD 239745 & 8 & $61 \pm 3$ & $8.22[1]$ & $7.58 \pm 0.07[3]$ & $8.57 \pm 0.05[7]$ & $7.20[1]$ & $5.80[1]$ & $7.16 \pm 0.12[3]$ \\
\hline HD 239748 & 6 & $66 \pm 3$ & $8.25[1]$ & $7.68 \pm 0.09[4]$ & $8.63 \pm 0.09[7]$ & $7.35[1]$ & $5.97[1]$ & $7.31 \pm 0.06[3]$ \\
\hline
\end{tabular}


Table 4. Non-LTE Abundances

\begin{tabular}{ccccccccc}
\hline Star & $\begin{array}{c}\xi \\
\left(\mathrm{kms}^{-1}\right)\end{array}$ & $\begin{array}{c}v \sin i \\
\left(\mathrm{kms}^{-1}\right)\end{array}$ & $\log \epsilon(\mathrm{C})$ & $\log \epsilon(\mathrm{N})$ & $\log \epsilon(\mathrm{O})$ & $\log \epsilon(\mathrm{Mg})$ & $\log \epsilon(\mathrm{Al})$ & $\log \epsilon(\mathrm{Si})$ \\
\hline HD 202347 & 6 & $119 \pm 6$ & - & $7.54 \pm 0.04$ & $8.54 \pm 0.12$ & 7.35 & 6.28 & $7.13 \pm 0.06$ \\
HD 205948 & 5 & $144 \pm 11$ & 8.35 & $7.35 \pm 0.05$ & $8.29 \pm 0.03$ & 7.05 & 6.18 & $6.95 \pm 0.13$ \\
HD 207951 & 5 & $87 \pm 2$ & 8.47 & $7.65 \pm 0.05$ & $8.72 \pm 0.10$ & - & - & $7.05 \pm 0.07$ \\
HD 209339 & 3.5 & $98 \pm 9$ & - & $7.34 \pm 0.03$ & $8.36 \pm 0.12$ & 7.38 & 6.07 & $7.44 \pm 0.03$ \\
HD 227696 & 10 & $120 \pm 5$ & - & $7.49 \pm 0.06$ & $8.60 \pm 0.16$ & - & - & $7.41 \pm 0.16$ \\
HD 228199 & 5 & $104 \pm 6$ & 8.55 & $7.56 \pm 0.16$ & $8.67 \pm 0.16$ & 7.78 & 6.13 & $7.57 \pm 0.03$ \\
HD 235618 & 9 & $100 \pm 3$ & 8.54 & 7.81 & $8.47 \pm 0.15$ & 7.55 & 5.94 & $7.73 \pm 0.02$ \\
HD 239681 & 9 & $142 \pm 11$ & 8.10 & $7.70 \pm 0.16$ & $8.48 \pm 0.17$ & 7.82 & 6.48 & $7.55 \pm 0.03$ \\
HD 239710 & 8 & $64 \pm 5$ & 8.50 & $7.57 \pm 0.08$ & $8.60 \pm 0.13$ & 7.63 & 5.96 & $7.71 \pm 0.05$ \\
HD 239729 & 5 & $99 \pm 8$ & - & $7.33 \pm 0.05$ & $8.28 \pm 0.10$ & 7.26 & 5.86 & $6.86 \pm 0.07$ \\
HD 239745 & 6 & $61 \pm 2$ & 8.38 & $7.51 \pm 0.13$ & $8.46 \pm 0.09$ & 7.25 & 5.77 & $7.08 \pm 0.19$ \\
HD 239748 & 5 & $68 \pm 4$ & 8.27 & $7.55 \pm 0.07$ & $8.54 \pm 0.08$ & 7.42 & 5.95 & $7.13 \pm 0.13$ \\
\hline
\end{tabular}


Table 5. Abundance Uncertainties.

\begin{tabular}{|c|c|c|c|}
\hline Ion & correction & HD 239710 & HD 239745 \\
\hline \multirow[t]{6}{*}{ C III } & $\delta\left(T_{e f f}\right)$ & -0.25 & -0.28 \\
\hline & $\delta(\log g)$ & +0.07 & +0.08 \\
\hline & $\delta(\xi)$ & 0.00 & -0.06 \\
\hline & $\delta(v \sin i)$ & +0.03 & +0.03 \\
\hline & $\delta($ continuum $)$ & +0.02 & +0.04 \\
\hline & $\delta_{t}$ & +0.26 & +0.30 \\
\hline \multirow[t]{6}{*}{ N II } & $\delta\left(T_{e f f}\right)$ & -0.10 & +0.05 \\
\hline & $\delta(\log g)$ & +0.03 & -0.01 \\
\hline & $\delta(\xi)$ & -0.05 & -0.01 \\
\hline & $\delta(v \sin i)$ & +0.02 & +0.01 \\
\hline & $\delta($ continuum $)$ & +0.02 & +0.03 \\
\hline & $\delta_{t}$ & +0.12 & +0.06 \\
\hline \multirow[t]{6}{*}{ O II } & $\delta\left(T_{e f f}\right)$ & -0.15 & -0.05 \\
\hline & $\delta(\log g)$ & +0.03 & +0.05 \\
\hline & $\delta(\xi)$ & -0.07 & -0.10 \\
\hline & $\delta(v \sin i)$ & +0.02 & +0.01 \\
\hline & $\delta($ continuum $)$ & +0.03 & +0.03 \\
\hline & $\delta_{t}$ & +0.17 & +0.13 \\
\hline \multirow[t]{6}{*}{$\mathrm{Mg}$ II } & $\delta\left(T_{e f f}\right)$ & +0.13 & +0.12 \\
\hline & $\delta(\log g)$ & -0.07 & -0.07 \\
\hline & $\delta(\xi)$ & -0.16 & -0.07 \\
\hline & $\delta(v \sin i)$ & +0.05 & +0.04 \\
\hline & $\delta($ continuum $)$ & +0.03 & +0.05 \\
\hline & $\delta_{t}$ & +0.22 & +0.17 \\
\hline \multirow[t]{6}{*}{$\mathrm{Al} \mathrm{III}$} & $\delta\left(T_{e f f}\right)$ & -0.01 & +0.03 \\
\hline & $\delta(\log g)$ & +0.02 & +0.03 \\
\hline & $\delta(\xi)$ & -0.04 & -0.04 \\
\hline & $\delta(v \sin i)$ & +0.01 & +0.01 \\
\hline & $\delta$ (continuum) & +0.01 & +0.02 \\
\hline & $\delta_{t}$ & +0.05 & +0.06 \\
\hline \multirow[t]{6}{*}{ Si III } & $\delta\left(T_{e f f}\right)$ & -0.17 & +0.04 \\
\hline & $\delta(\log g)$ & +0.04 & -0.02 \\
\hline & $\delta(\xi)$ & -0.17 & -0.14 \\
\hline & $\delta(v \sin i)$ & +0.02 & +0.02 \\
\hline & $\delta($ continuum $)$ & +0.02 & +0.03 \\
\hline & $\delta_{t}$ & +0.24 & +0.15 \\
\hline
\end{tabular}




\section{Figure Captions}

Fig 1. Variation of LTE oxygen abundance with the microturbulence velocity for the sample star HD 239745. The adopted solution is $\xi=8 \mathrm{kms}^{-1}$ that corresponds to $\log \epsilon(O)=8.57 \pm 0.05$.

Fig 2. Comparison between observed and non-LTE synthetic profiles for one of the spectral regions containing O II lines. The observed spectrum is for the target star HD239748 and the synthetic profiles were calculated for different sets of parameters. Top: on the left panel we show synthetic profiles calculated for five values of oxygen abundances (indicated in the figure). The best fit oxygen abundance is derived for $\log \epsilon(O)=8.42$ (represented by the solid line). In the right panel we present the variation of $\chi^{2}$ as a function of oxygen abundance. Bottom: same for different values of $v \sin i$ varying from 50 to $80 \mathrm{kms}^{-1}$.

Fig 3. Non-LTE abundances as a function of adopted effective temperatures. The derived abundances show almost no trend with effective temperature and are, in general, sub-solar. There are some stars that have solar, and in some cases, above solar abundances of $\mathrm{C}, \mathrm{O}, \mathrm{Mg}, \mathrm{Al}$, and Si. The reference solar abundances (dotted lines) are from Grevesse, Noels \& Sauval (1996) for all elements except for oxygen for which we adopt an average abundance value between the recent studies by Prieto et al. (2001) and Holweger (2001).

Fig 4. The non-LTE correction $\Delta$ (LTE - non-LTE) as a function of effective temperature.

Fig 5. The derived stellar parameters $T_{\text {eff }}$ and $\log g$ are plotted in the top panel to form a modified HR-diagram for Cep OB2 stars. Members of the young cluster Tr 37 are plotted as the open circles, and all other association members are plotted as filled circles. The Tr 37 members should be generally younger and this is found to be the case. Solid curves are stellar models from Schaller et al. (1992) for 7, 9, 12, 15 and $20 \mathrm{M}_{\odot}$ models, with the dashed line denoting the ZAMS. Two fairly well-defined, differing age subgroups are identified (based upon the Schaller et al. models, the age difference is $\sim 2-5 \mathrm{Myr})$. The middle panel shows $\log (\mathrm{N} / \mathrm{O})$ abundance ratios versus surface gravity, as the surface gravity of a star will initially decrease as it evolves off of the main sequence. Note that the two highest N/O-ratio stars have the lowest surface gravities (these two are the most highly evolved of the $\sim 15 \mathrm{M}_{\odot}$ stars). The dotted line is the average $\log (\mathrm{N} / \mathrm{O})=-0.99$ of the other Cep OB2 stars. The bottom panel plots $\log (\mathrm{N} / \mathrm{O})$ versus $v \sin i$ and shows that the two N-rich evolved stars are also two of the most rapidly rotating ones: rotationally induced mixing of $\mathrm{CN}$-cycle material to the surface is one viable explanation for the increased $\mathrm{N} / \mathrm{O}$ ratios. 


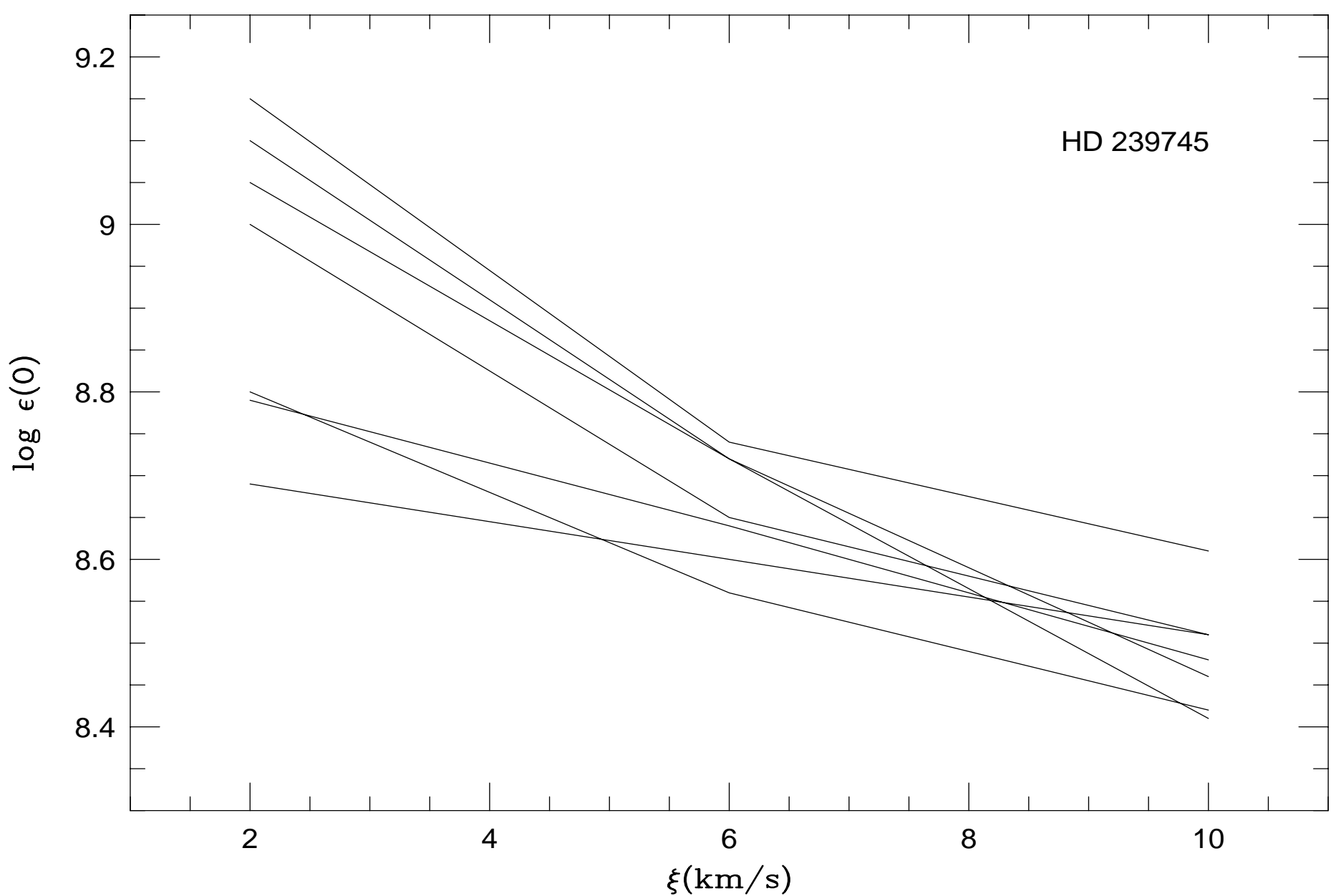



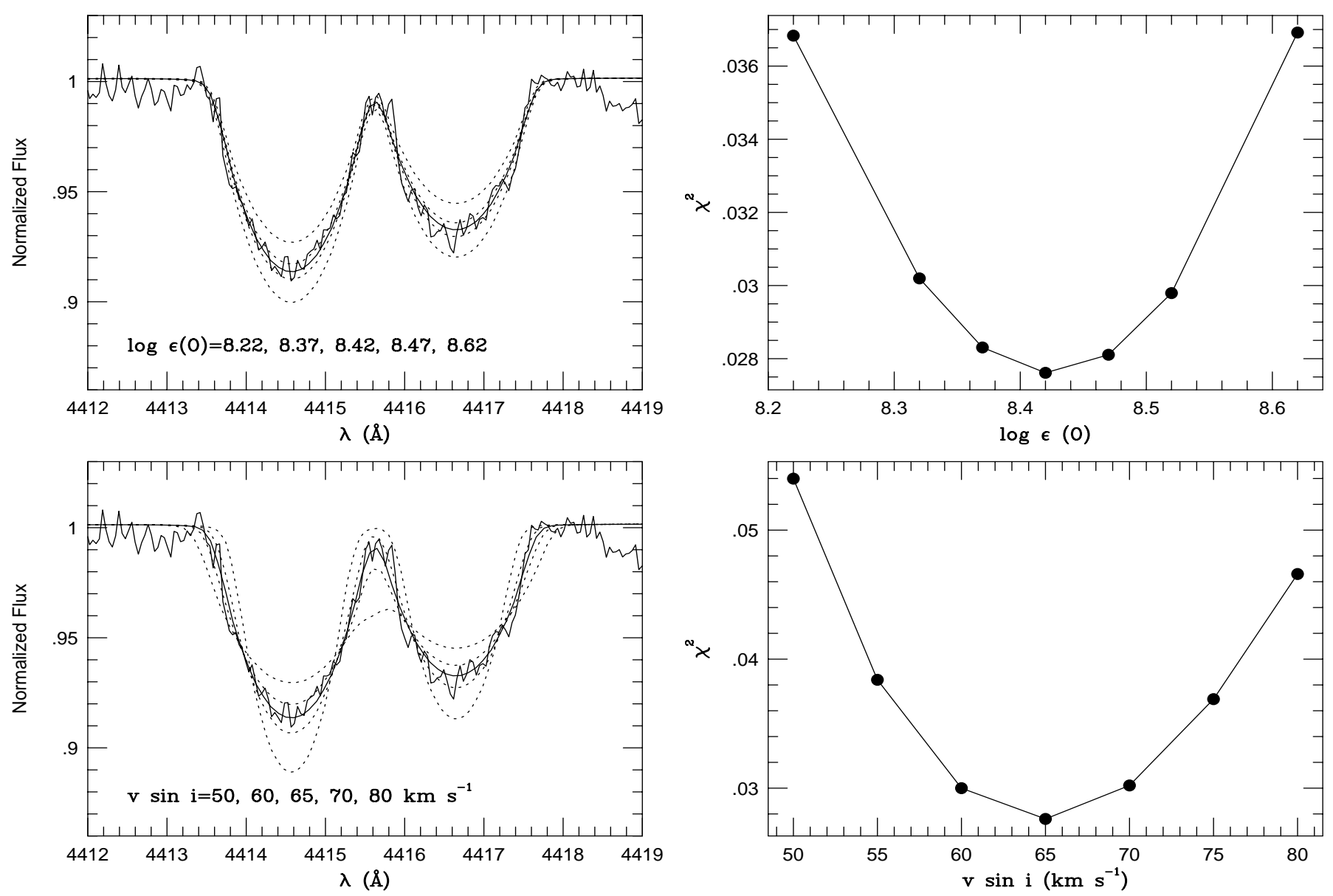


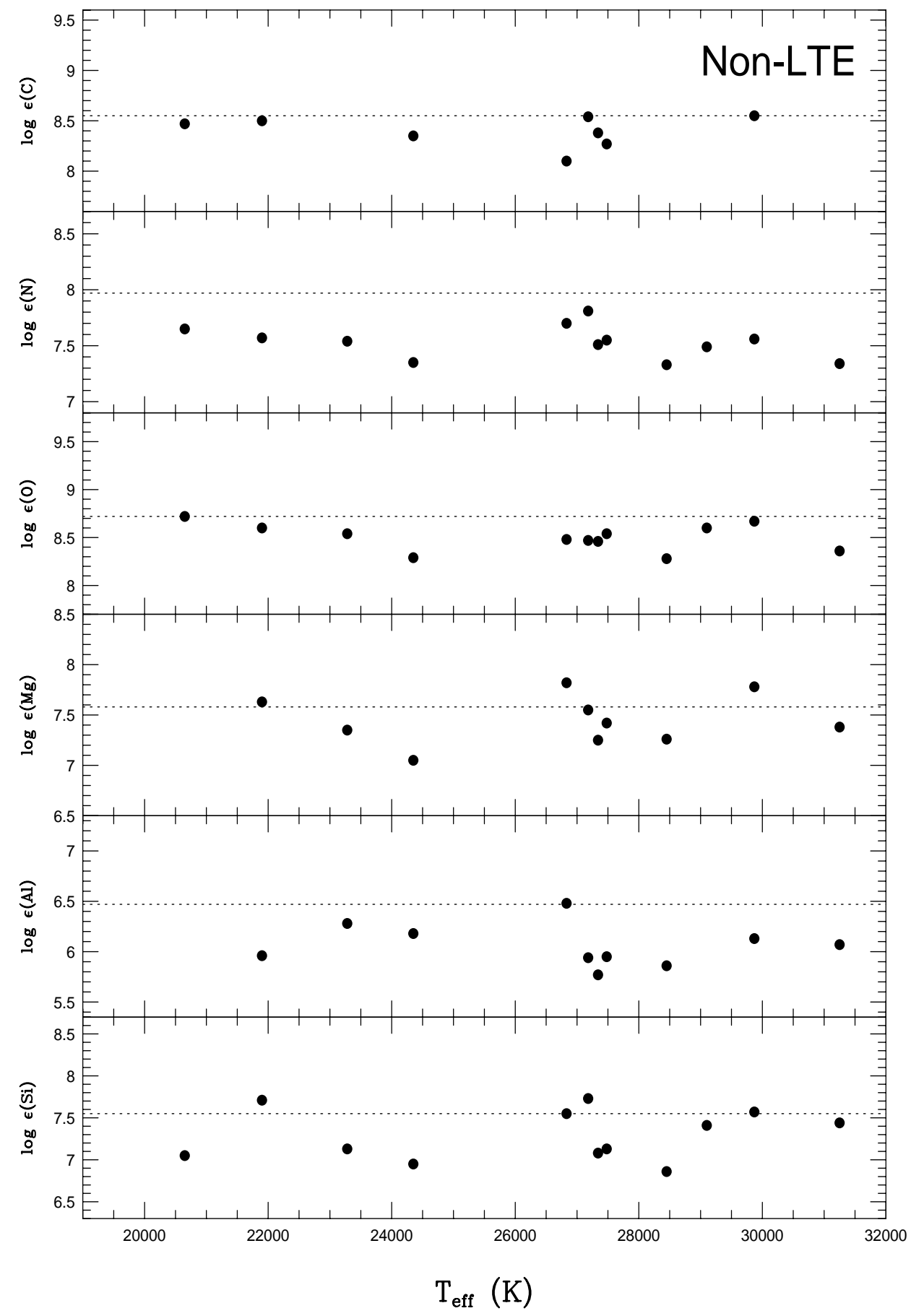

Fig. 3.- 


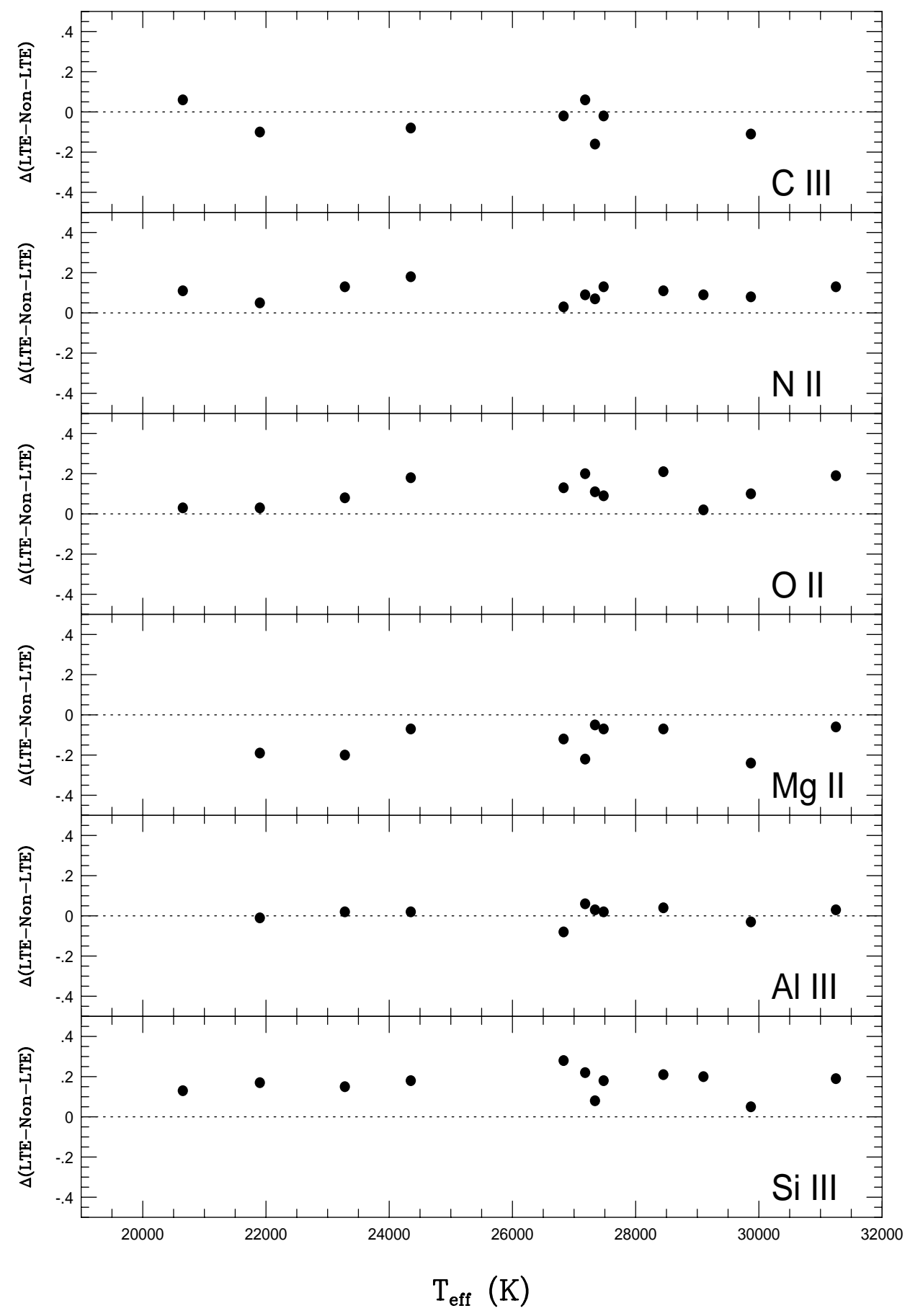

Fig. 4.- 

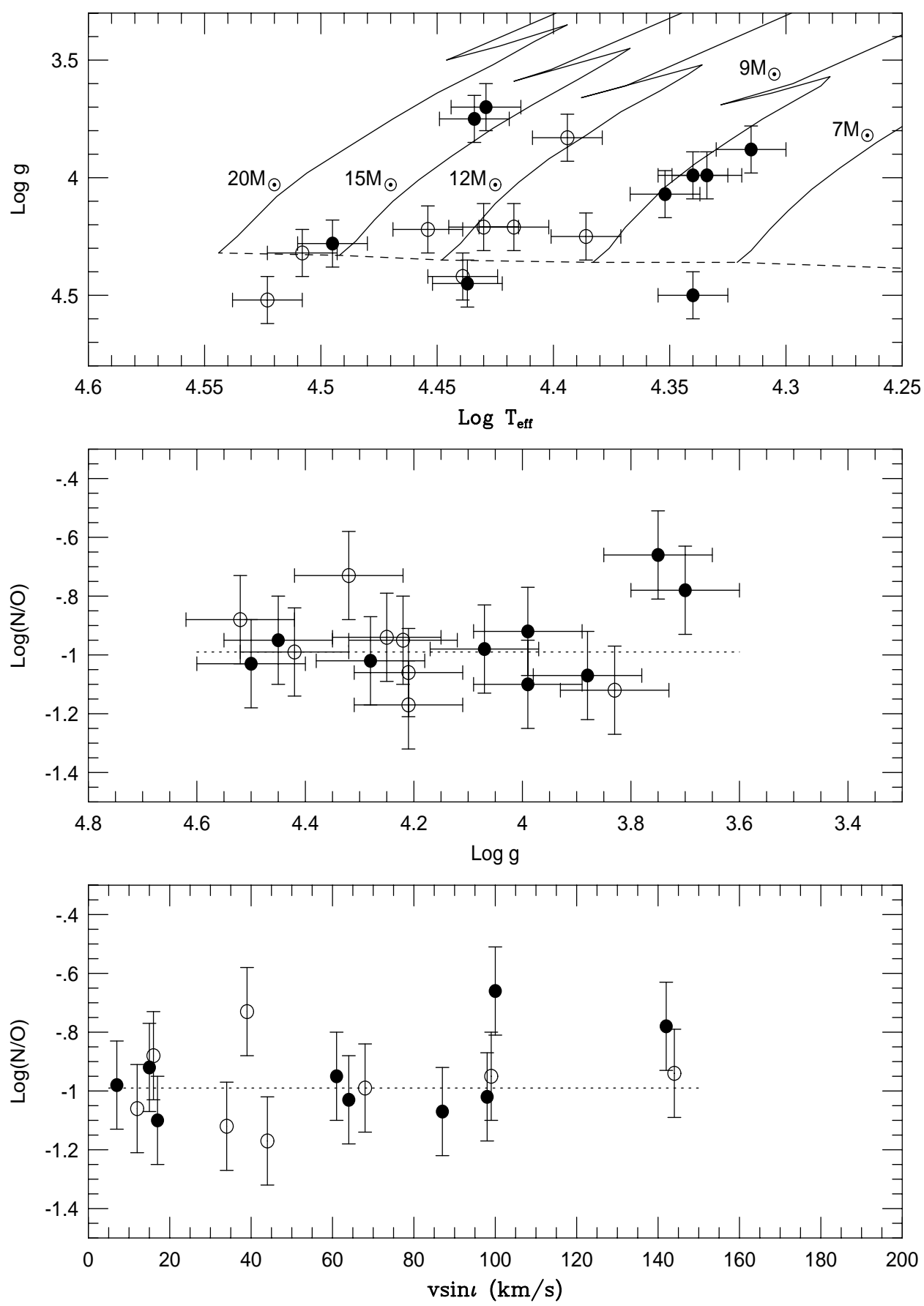

Fig. 5.- 